\title{
Subcutaneous Immunoglobulin Replacement Therapy in Patients with Primary Immunodeficiency in Routine Clinical Practice: The VISPO Prospective Multicenter Study
}

\author{
Alessandra Vultaggio • Chiara Azzari · Cinzia Milito • Andrea Finocchi · \\ Claudia Toppino $\cdot$ Giuseppe Spadaro $\cdot$ Antonino Trizzino $\cdot$ Martire Baldassarre • \\ Roberto Paganelli $\cdot$ Viviana Moschese $\cdot$ Annarosa Soresina $\cdot$ Andrea Matucci
}

Published online: 12 February 2015

(c) The Author(s) 2015. This article is published with open access at Springerlink.com

\begin{abstract}
Background and Objectives Subcutaneous immunoglobulin (SCIG) therapy is becoming increasingly popular as self-administration is possible because intravenous access is unnecessary, and there is a lower frequency of systemic adverse events. The aim of this study was to evaluate the shifting from intravenous immunoglobulins (IVIGs) replacement therapy to SCIG in patients with primary immunodeficiency (PID) in a routine real-life situation.

Methods In a multicenter prospective observational study, we enrolled 50 patients suffering from PID who were monitored for 24 months; 44 patients switched from IVIG and six from different SCIG preparations. The study
\end{abstract}

\footnotetext{
A. Vultaggio · A. Matucci $(\bowtie)$

Immunoallergology Unit, Department of Biomedicine,

Policlinico di Careggi, Viale Morgagni, 85,

50134 Florence, Italy

e-mail: andrea.matucci@unifi.it

C. Azzari

Immunology Department, Anna Meyer Children's Hospital,

Florence, Italy

C. Milito

Department of Clinical Immunology, University of Rome

La Sapienza, Rome, Italy

A. Finocchi

Unit of Immunology and Infectious Disease,

University-Hospital Pediatric Department, Bambino Gesù

Children Hospital, Rome, Italy

C. Toppino

Department of Pediatrics, University of Turin, Turin, Italy

G. Spadaro

Department of Internal Medicine, Cardiovascular sciences and Immunology, University of Naples Federico II, Naples, Italy
}

preparation (human IgG $16 \%$, Vivaglobin ${ }^{\circledR}$, CSL Behring $\mathrm{GmbH}$, Germany) was subcutaneously infused weekly (maximum volume $15 \mathrm{~mL} / \mathrm{site}$; maximum infusion rate $22 \mathrm{~mL} / \mathrm{h}$ ). The study endpoints were: annual rate of severe bacterial infections (SBIs), local adverse reactions, quality of life, days off school/work, and days of hospitalization. Results Thirty-three of 39 (84.6\%) patients who completed the study experienced an infection or signs thereof. Only five SBIs were observed, corresponding to an annual rate of 0.056 episodes per patient in 44 subjects [intentionto-treat (ITT) population]. A significant decrease in both days of hospitalization $(1.93 \pm 4.08$ vs. $0.64 \pm 2.94)$ and days off school/work (15.27 \pm 23.17 vs. $2.26 \pm 4.45)$ was

\author{
A. Trizzino \\ Department of Pediatric Hematology and Oncology, ARNAS \\ Civico, Di Cristina and Benfratelli Hospital, Palermo, Italy \\ M. Baldassarre \\ Department of Biomedical Sciences and Human Oncology, \\ Unit of Pediatrics Policlinico, University of Bari, Bari, Italy \\ R. Paganelli \\ Department of Medicine and Sciences of Aging, University \\ G.d'Annunzio, Chieti, Italy \\ V. Moschese \\ Department of Pediatrics, University of Rome Tor Vergata, \\ Rome, Italy \\ A. Soresina \\ Department of Pediatrics, Institute of Molecular Medicine \\ “A. Nocivelli", University of Brescia, Brescia, Italy
}


recorded at 24 months. Local reactions were observed in $14 / 50(28 \%)$ patients, mainly consisting of skin manifestations at the injection site. Only three $(6.8 \%)$ patients discontinued due to infusion site reactions. In patients shifting from IVIG to SCIG, the total mean score of Life Quality Index (LQI) improved from $76.9 \pm 16.8$ to $90.7 \pm 11.6(P<0.01)$ at 6 months; there was an improvement also in the overall patients' evaluation.

Conclusions A total of $93.2 \%$ patients tolerated the new route of administration and reported a significant improvement in their LQI. Our results from a routine clinical practice in a real-life population are consistent with those of phase III clinical studies.

\section{Key Points}

SCIG therapy is effective and well tolerated in real-life situations.

SCIG therapy leads to a less interference with private life and work.

SCIG therapy increases the treatment satisfaction referred by PID patients.

\section{Introduction}

Primary immunodeficiency (PID) disorders that predispose patients to recurrent respiratory, skin, and gastrointestinal infections, require immunoglobulin (Ig) replacement therapy. Ig replacement therapy is beneficial although the optimal immunoglobulin $\mathrm{G}$ ( $\operatorname{IgG}$ ) trough level to be maintained over time in order to minimize risks of infections has not yet been established [1]. The most common method of administration of $\mathrm{Ig}$ is via the intravenous (IVIG) route, although today there are different options, for example the subcutaneous route. Subcutaneous Ig (SCIG) replacement offers many advantages, such as the maintenance of stable serum IgG levels in comparison to IVIG, hospital-free setting, improvement of patients' quality of life (QoL), better tolerability, and low incidence of side effects [2]. Dosing of SCIG in PID patients should be based on their current dose of IVIG as well as their serum trough levels. The weekly subcutaneous dose should be calculated by dividing by four the current monthly intravenous dose corresponding to the new treatment interval $[3,4]$.

Our prospective, observational, 24-month, multicenter study was designed to verify whether the tolerability profile of the SCIG administration shown during phase III pivotal

trials $[3,5]$ carried out on a selected population, was confirmed during the subcutaneous administration of Vivaglobin ${ }^{\circledR}$ (CSL Behring GmbH, Germany) in PID patients, in a routine clinical real-life setting. Indeed, Gardulf et al. [3] determined the annualized rate of severe infections as 0.04 episodes/patient; moreover, $28(1 \%)$ systemic adverse reactions occurred, none of them severe. Local tissue reactions declined over time, this being particularly evident after 8-10 weeks. Ochs et al. [5] recorded two patients $(4 \%)$ who reported one severe bacterial infection each (pneumonia), with an annual rate of 0.04 per patientyear. The most frequent treatment-related adverse event was infusion-site reaction (91\% of patients), which was predominantly mild or moderate and decreased over time.

We also collected efficacy data and the impact, if any, on the patients' QoL.

\section{Patients and Methods}

\subsection{Study Design and Patients}

The study described herein is a prospective, 24-month, observational, multicenter study. Fifty patients (31 males and 19 females) with PID according to the definitions provided by the European Society for Immunodeficiencies (ESID), [32 Common Variable Immunodeficiency-CVID, $10 \mathrm{X}$-linked agammaglobulinemia (XLA), 8 others], were enrolled in 11 study sites in Italy. Inclusion of patients was decided according to the possibility and willingness to begin using the SCIG therapy, as assessed by the local trialist. Table 1 shows demographic and baseline data. The intention-to-treat (ITT) population (44 subjects) was defined as subjects with baseline data, who completed at least one follow-up. Thirty-nine patients were considered perprotocol (i.e., subjects who completed the 24-month observation). Safety population included 50 patients. For QoL assessment, the participants were divided into two groups according to age: Group A (older than 14 years, $n=43$ ), Group B (14 years or younger, $n=7$ ). Follow-up visits were at 6,12 and 24 months after study initiation. The study was performed in accordance with the International Conference on Harmonization, Good Clinical Practice guidelines and the Declaration of Helsinki, and received approval by the local Institutional Review Boards. All patients and/or carers provided their written, informed consent, including also the anonymous disclosure of findings.

\subsection{Subcutaneous Immunoglobulin}

Subcutaneous immunoglobulin (SCIG) was given as a weekly infusion by using a liquid pasteurized, polyvalent, 
Table 1 Demographic baseline characteristics of patients

\begin{tabular}{ll}
\hline Parameters & Values \\
\hline Patients enrolled, $n^{\mathrm{a}}$ & 50 \\
Group A (older than 14 years), $n$ & 43 \\
Group B (up to 14 years), $n$ & 7 \\
Age, years, mean $\pm \mathrm{SD}$ & $31.7 \pm 15.7$ \\
ITT patients, $n(\%)^{\mathrm{b}}$ & $44(88 \%)$ \\
Per protocol patients, $n^{\mathrm{c}}$ & 39 \\
Primary immunodeficiency diagnosis & \\
Common variable immunodeficiency, $n(\%)$ & $32(64 \%)$ \\
X-linked agammaglobulinemia, $n(\%)$ & $10(20 \%)$ \\
Others, $n$ (\%) & $8(16 \%)$ \\
Previous Ig treatment & $44(88 \%)$ \\
Intravenous, $n$ (\%) & $6(12 \%)$ \\
Subcutaneous, $n(\%)$ & $635 \pm 242.8$ \\
Baseline serum trough IgG levels, mg/dL, mean \pm SD & \\
\hline SD standard deviation, ITT intention-to-treat, Ig immunoglobulin \\
a Safety population \\
b Patients with baseline data who completed at least one follow-up \\
c Patients completing the 24-month observation period
\end{tabular}

human $16 \%$ preparation $(160 \mathrm{mg} / \mathrm{mL})\left(\right.$ Vivaglobin $^{\circledR}, \mathrm{CSL}$ Behring $\mathrm{GmbH}$, Germany). The patients maintained the total monthly dose of the previous Ig replacement therapy, split into four weekly doses. Serum IgG trough levels were monitored. The preparation was administered subcutaneously using infusion pumps in the abdomen or thigh. The maximum volume per injection site was $15 \mathrm{~mL}$ and the maximum infusion rate per site of $22 \mathrm{~mL} / \mathrm{h}$. Patients switching to the study therapy were trained and supervised weekly at the local hospital before starting self-infusions at home. The carers either infused their children or supervised the treatment. Patients already on a different SCIG treatment continued with their established infusion technique. No premedication was given.

\subsection{Primary and Secondary Endpoints}

\subsubsection{Efficacy and Safety Assessment}

The primary endpoint for efficacy was the annual rate of severe bacterial infections (SBIs) per patient, as defined by the FDA (Food and Drug Administration), including bacterial pneumonia, bacteremia/septicemia, osteomyelitis/ septic arthritis, bacterial meningitis, and visceral abscess [6]. The diagnosis of SBIs was performed according to standard medical procedures, such as physical examination, laboratory tests, bacterial cultures and imaging. Secondary end-points were reported by each patient in a specific diary, including number of days off school/work, days of hospitalization due to infections as well as local and systemic tolerability, which was assessed by patients and then reviewed by the investigator during the follow-up visits.

\subsubsection{Quality of Life Assessment}

The acceptability of SCIG and its impact on overall QoL were evaluated by using standardized, validated healthrelated QoL (HRQoL) questionnaires, such as the Child Health Questionnaire-Parental Form 50 (CHQ-PF50), the Short Form 36 (SF-36), and the Life Quality Index (LQI). The subjects and/or carers completed these questionnaires at baseline and at the follow-up visits $(6,12$, and 24 months). The CHQ-PF50 was used for children and young adolescents up to 14 years of age and was, for the most part, completed by carers. The assessment evaluates 15 concepts and focuses on the physical and psychosocial functioning and well-being of the child and his or her family. The Short Form 36 (SF-36) was used for adults, and we referred to 35 items divided into eight subscales: physical function (10 items), role-physical (4 items), body pain ( 2 items), general health (5 items), vitality (4 items), social function (2 items), role-emotional (3 items), and mental health (5 items). Higher scores indicated a better HRQoL in both questionnaires. The LQI examines the respondent's perceptions of the impact of the $\mathrm{IgG}$ treatment on daily activities. The LQI is worded without referencing home or clinic-based settings [7]. Patients' perception of their general health was also evaluated by the subjects using a 100-mm Visual Analogue Scale (VAS) ranging from 0 (worst) to 100 (best). Data were also collected regarding days off school/work in the 12 months prior to enrollment and recorded during the observation period, in patient's diaries.

\subsection{Statistical Methodology}

Since the number of subjects enrolled in phase III multicenter international trials ranged between $20-40$, an evaluable population of 30 patients was considered an adequate sample for the present 1-year, single-country study. For subjects for whom data were available for the entire study period, we adopted the Friedman's non-parametric test for repeated measures. Descriptive statistics was also applied. No post-hoc analysis was performed. Statistical significance was defined by $P$ values $<0.05$. BMDP Dynamic 2009 v.8.2. was used for calculations [8].

\section{Results}

The recruitment period was 12 months in each center. The recruitment started in October 2008 and was completed in 
Fig. 1 Days off work/school in PID patients during the 24-month observation period. Mean (SD) values of days off work or school for the ITT population at 6,12 , and 24 months from the switch to SCIG. Data obtained from both descriptive analysis and Friedman's test, are reported. Retrospective baseline data are relating to the 12 months prior to enrolling $(n=41)$. PID primary immunodeficiency, ITT intention-to-treat, $S C I G$ subcutaneous immunoglobulin

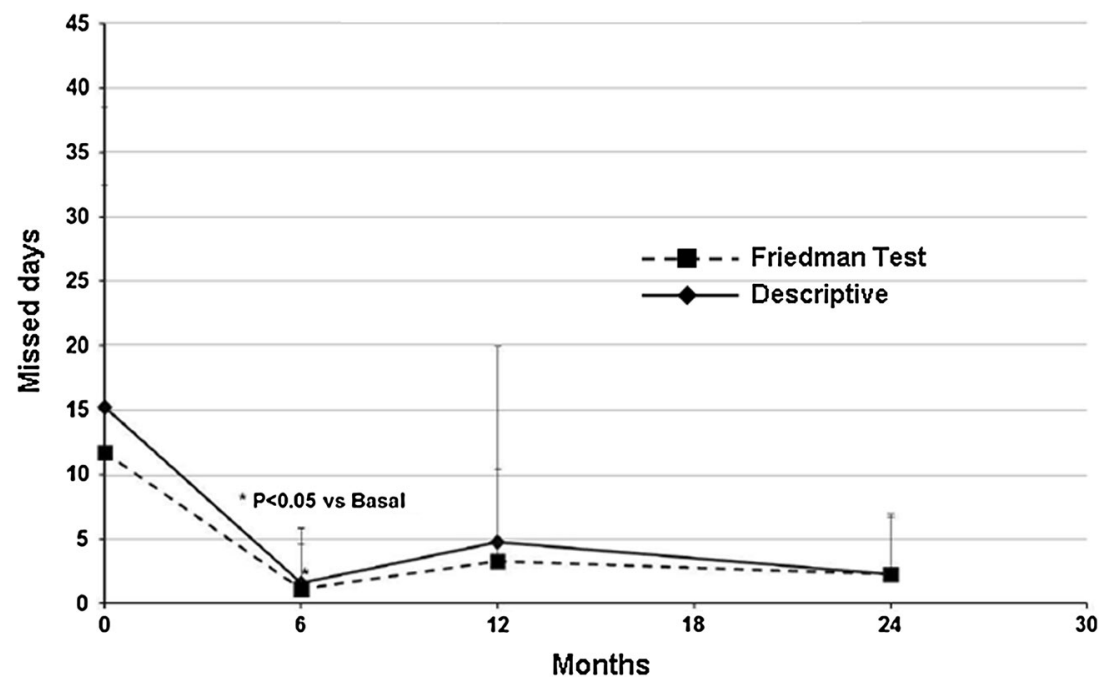

March 2011, for an overall recruitment period of about 29 months.

Patients and/or carers required four to seven training sessions to familiarize themselves with the subcutaneous infusion.

A total of 39 patients completed the 24-month observation period.

Trough mean serum levels of IgG antibodies increased slightly during SCIG treatment from $635 \pm 242.8$ to $671.5 \pm 217.5 \mathrm{mg} / \mathrm{dL}(\mathrm{ns})$.

\subsection{Severe Bacterial Infections and Infectious Complications}

Five SBIs (all bacterial pneumonia) were observed in five patients, corresponding to an infection rate of $0.056 \mathrm{per}$ patient/year (ITT population).

Thirty-three out of $39(84.6 \%)$ evaluable per protocol patients experienced at least one infection or possible signs or symptoms of infection during the 24-month follow-up period. Sinusitis (52\%), bronchitis (46\%), gastrointestinal (34\%) and urinary infections (4\%) were most frequently reported. Days off work/school decreased significantly during the SCIG therapy (baseline data on 41 subjects) (Fig. 1). A small non-significant reduction was also recorded in the number of days in hospital (1.93 \pm 4.08 vs. $0.64 \pm 2.94$ ), which was lower during the use of SCIG.

\subsection{Improvement in the Quality Of Life}

No significant improvement during the SCIG treatment was observed in QoL assessment at 6,12 and 24 months in both Group A $(n=44)$ and Group B $(n=5)$ compared to baseline values (Fig. 2a, b). However, for both groups the total mean LQI score improved significantly from $76.88 \pm 16.76$ to $90.67 \pm 11.64(P<0.01)$ at 6 months, and the improvement was sustained over time (Fig. 3). Indeed, the patients' overall evaluation of Ig therapy improved significantly $(P<0.05)$ in the 37 subjects shifting from IVIG to SCIG, when comparing the baseline with the final evaluation at the end of the 24-month observation period. Based on the 100-mm VAS scale, the subjective assessment of the general health status associated with SCIG therapy showed a slightly non-significant increase compared to baseline values $(65.27 \pm 17.83 \quad$ vs. $68.26 \pm 19.78)$.

\subsection{Safety: Local and Systemic Adverse Reactions}

Local reactions and symptoms were observed in 14 (28\%) out of the total of 50 enrolled patients, representing the safety population. Skin manifestations (erythema, edema, itching and pain) at the injection site were the most common findings. They were observed in 18, 20 and $14 \%$ of patients at 6,12 and 24 months, respectively, thus showing a slight tendency to decrease, which confirms previous findings [4]. Systemic symptoms included nausea, fever and diarrhea and their overall incidence remained stable over time (Fig. 4). No severe systemic adverse reactions were recorded. Three patients $(7 \%)$ discontinued SCIG therapy due to infusion-site reactions characterized by local pain. No remarkable changes in hematological or other laboratory parameters were observed during the study, and no product-related, virus-safety issues were found.

\subsection{Drop Outs}

Eleven out of $50(22 \%)$ patients discontinued the study. Six patients withdrew at the 6-month follow-up, two patients at month 12 and three patients at month 24. Reasons for patient discontinuation were absence from follow-ups $(n=3,27.3 \%)$, change of Ig preparations $(n=4$, 
Fig. 2 Results of HRQoL questionnaires. The Short Form 36 (SF-36) was used for Group A, older than 14 years $(n=44)$. The Child Health

Questionnaire-Parental Form 50 (CHQ-PF50) was used for

Group B, 14 years or younger, and answered by the carers $(n=5) . H R Q o L$ health-related quality of life

Fig. 3 Treatment satisfaction assessed by Life Quality Index scale. Data are given as selfreported summary scores at baseline, at 6, 12 and 24 months (Group A, $n=44$; Group B, $n=5$ ). Data obtained from both descriptive analysis and Friedman's test, are reported. A maximum summary score of 105 indicates the highest possible satisfaction with the treatment on factors such as independence, therapy convenience, social/work/ school activities, and health and travel costs
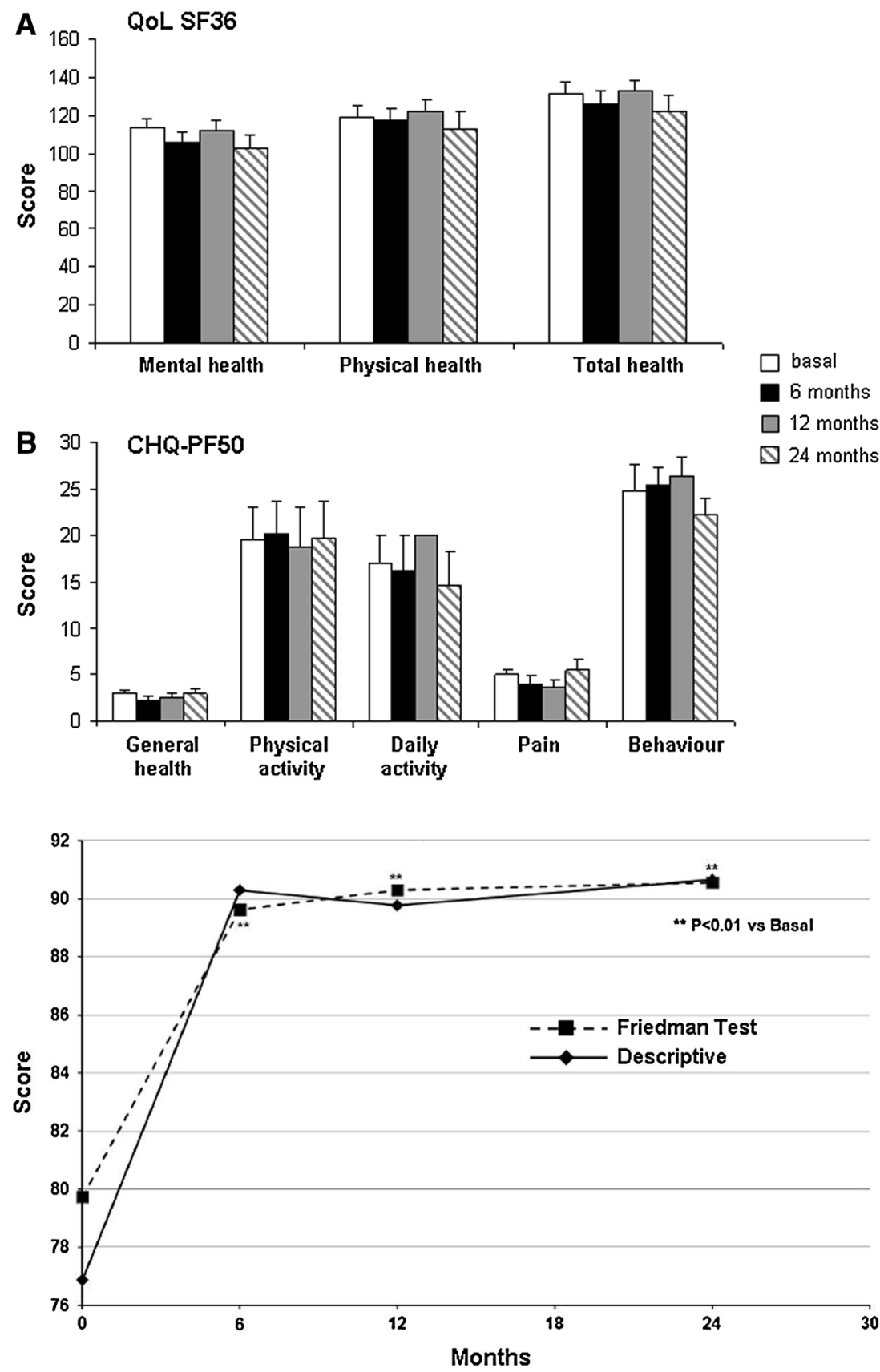

$36.3 \%)$, no compliance $(n=1,9.0 \%)$ and adverse reactions $(n=3,27.3 \%)$.

\section{Discussion}

PID disorders that predispose patients to recurrent infections require Ig replacement therapy. Intravenous Ig administration has been established as a safe and efficacious treatment in patients suffering from PID [1]. However, subcutaneous administration of Ig provides a better treatment experience for these patients by allowing home therapy, thus avoiding hospitalization, and maintaining stable IgG serum levels [3, 9]. In addition, for most patients, self-administration results in improved convenience, such as fewer absences from work and school.

Overall, the results of the VISPO study showed that Vivaglobin ${ }^{\circledR}$ was effective and well tolerated in the reallife routine population performing self-administration at home.

It is known that patients suffering from PID may experience a high rate of four or more severe respiratory infections/year without Ig replacement therapy. In our experience, SCIG treatment has proven to be effective as 
Fig. 4 Treatment-related most common adverse reactions. Skin reactions include redness, edema, itching and pain at the injection site $(n=50)$

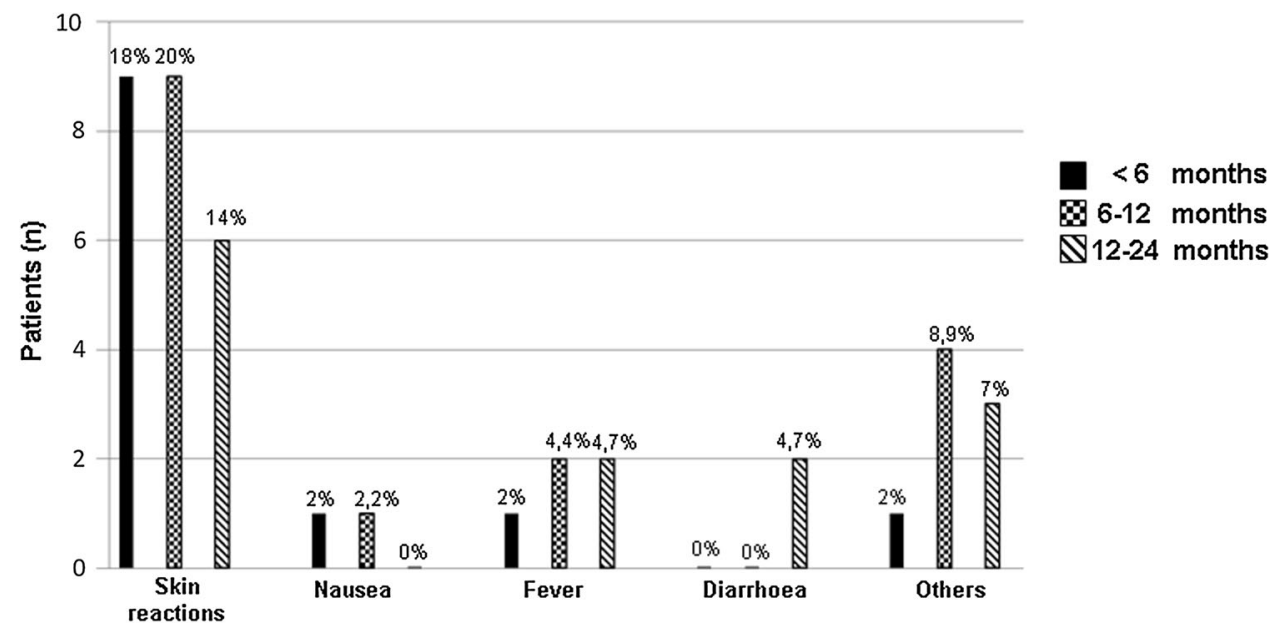

shown by a low annualized rate of SBI $(0.06 \mathrm{SBI} /$ patient/ year) recorded during the 24-month follow-up period. Our data are in line with previously published results obtained in a prospective, multicenter study [10].

In the follow-up period of our study, minor infections such as sinusitis, bronchitis, and gastrointestinal infections were recorded but did not result in days off work/school. Moreover, a significant reduction in days off work/school was observed during the SCIG treatment. This is strictly related to the impact of the treatment on QoL. Group A (patients older than 14 years) did not report any significant improvement of SF-36 scores (mental and physical health) at 6, 12 and 24 months, compared to baseline. Similarly, the smaller Group B (patients 14 years or younger) did not report any improvement of CHQ-PF50 scales. These results appear in contrast to those reported in other studies performed in Europe and North America, which found significant improvement of HRQoL in patients suffering from PID treated with SCIG $[11,12]$. However, the LQI index, which records the respondent's perceptions of the impact of SCIG-treatment on daily activities, was better tailored to collect the potential advantages of the home-based SCIG therapy and the reduced dependency from hospital. In fact, in both groups, the total mean LQI score significantly improved at six months, and was maintained over time. In addition, the increase in treatment satisfaction, referred by patients shifting from IVIG to SCIG, reflects and confirms the improvement of LQI induced by the subcutaneous therapy.

However, from our study it is not possible to define whether the improvement of LQI is primarily related to the use of the SCIG therapy itself, the switch to home treatments, or both. The LQI improvement showed that SCIG therapy allowed greater independence, less impact on work/school and social activities, and treatment flexibility with less disruption of daily activities.
Since SCIG therapy could minimize the impact of chronic diseases, such as PID, on patients' and families' active lives without compromising therapy, it could be particularly suitable both for children and working people.

Other advantages of SCIG include its lower rate of adverse reactions compared with IVIG [3, 8, 9].

The VISPO study confirmed that SCIG therapy is well tolerated, with no apparent safety concerns. In fact, Vivaglobin ${ }^{\circledR}$ treatment showed good tolerability with regard to both local injection-site reactions and systemic adverse reactions. It is important to note that in the VISPO population, systemic symptoms included fever, nausea, and diarrhea, but no severe systemic reaction was recorded. However, three subjects discontinued the treatment due to local pain caused by the subcutaneous injection, an observation which is consistent with previous international clinical trials [3, 4]. The safety profile of SCIG emerging from phase III trials-even if direct comparative studies have not been performed-led health authorities to authorize SCIGs for home treatment, whereas IVIGs, in most of the countries, are authorized for hospital use only $[3,4]$.

The rate of withdrawal due to injection-site reactions can be minimized by patient education on the correct technique of administration, adherence to the infusion schedule, as well as by informing patients that possible local reactions should be expected. It should be emphasized that none of our patients received premedication.

Strictly related to the SCIG home treatment is the fact that life-threatening anaphylactic shock, has not been recorded so far. In fact, the administration of small doses using the subcutaneous route favored gradual absorption and decreased the incidence of hypersensitivity immediate reactions $[3,8]$. Moreover, the safety profile of SCIG is confirmed by the clinical observations that it can be administered to patients with anti-IgA antibodies, and patients who are potentially at risk for anaphylactic reactions during IVIG therapy [13]. In addition, in these patients, 
SCIG may even lead to a decrease of anti-IgA antibodies [5].

Finally, considering the issue regarding venous access for IVIG, the subcutaneous route of administration, is an additional benefit particularly in young subjects. Therapy with SCIG can minimize the use of corticosteroids and antihistamines prior to infusions, which is an additional benefit taking into account the life-long treatment plan.

\section{Conclusion}

In conclusion, the VISPO study performed in a real-life setting, confirmed the preliminary results of phase III trials with regard to the additional benefits of the SCIG therapy, particularly the improved systemic tolerability that allows self-infusion at home leading to better management of the disease and less interference with private life and work.

Acknowledgments This study was made possible thanks to the support of CSL Behring S.p.A. Italy. Andrea Matucci received fee for to be on the board of the study and for writing the manuscript. All other authors declare no conflicts of interest that are directly relevant to the content of this study.

Open Access This article is distributed under the terms of the Creative Commons Attribution Noncommercial License which permits any noncommercial use, distribution, and reproduction in any medium, provided the original author(s) and the source are credited.

\section{References}

1. Busse PJ, Razvi S, Cunningham-Rundles C. Efficacy of intravenous immunoglobulin in the prevention of pneumonia in patients with common variable immunodeficiency. J Allergy Clin Immunol. 2002;109:1001-4.

2. Shapiro RS. Why i use subcutaneous immunoglobulin (SCIG). J Clin Immunol. 2013;33(sup 12):S95-8.

3. Gardulf A, Nicolay U, Asensio O, Bernatowska E, Böck A, Carvalho BC, Granert C, Haag S, Hernández D, Kiessling P, Kus J, Pons J, Niehues T, Schmidt S, Schulze I, Borte M. Rapid subcutaneous $\mathrm{IgG}$ replacement therapy is effective and safe in children and adults with primary immunodeficiencies-a prospective, multi-national study. J Clin Immunol. 2006;26:177-85.

4. Desai SH, Chouksey A, Poll J, Berger M. A pilot study of equal doses of $10 \%$ IGIV given intravenously or subcutaneously. J Allergy Clin Immunol. 2009;124:854-6.

5. Ochs HD, Gupta S, Kiessling P, Nicolay U, Berger M, Subcutaneous IgG Study Group. Safety and efficacy of self-administered subcutaneous immunoglobulin in patients with primary immunodeficiency diseases. J Clin Immunol. 2006;26:265-73.

6. US Food and Drug Administration (FDA). FDA Guidance for industry: safety, efficacy, and pharmacokinetic studies to support marketing of immune globulin intravenous (human) as replacement therapy for primary humoral immunodeficiency. 2008. Available at: http://www.fda.gov/downloads/BiologicsBlood Vaccines/GuidanceComplianceRegulatoryInformation/Guidances/ Blood/ucm078526.pdf. Accessed 10 Feb 2015.

7. Nicolay U, Haag S, Eichmann F, Herget S, Spruck D, Gardulf A. Measuring treatment satisfaction in patients with primary immunodeficiency diseases receiving lifelong immunoglobulin replacement therapy. Qual Life Res. 2005;14:1683-91.

8. Armitage P, Berry G, Matthews JNS. Statistical methods in medical research. 4th ed. Blackwell Science; 2002.

9. Chapel HM, Spickett GP, Ericson D, Engl W, Eibl MM, Bjorkander J. The comparison of the efficacy and safety of intravenous versus subcutaneous immunoglobulin replacement therapy. J Clin Immunol. 2000;20:94-100.

10. Borte M, Quinti I, Soresina A, Fernández-Cruz E, Ritchie B, Schmidt DS, McCusker C. Efficacy and safety of subcutaneous vivaglobin ${ }^{\circledR}$ replacement therapy in previously untreated patients with primary immunodeficiency: a prospective, multicenter study. J Clin Immunol. 2011;31:952-61.

11. Gardulf A, Nicolay U, Math D, Asensio O, Bernatowska E, Böck A, Costa-Carvalho BT, Granert C, Haag S, Hernández D, Kiessling P, Kus J, Matamoros N, Niehues T, Schmidt S, Schulze I, Borte M. Children and adults with primary antibody deficiencies gain quality of life by subcutaneous $\operatorname{IgG}$ self-infusions at home. J Allergy Clin Immunol. 2004;114:936-42.

12. Nicolay U, Kiessling P, Berger M, Gupta S, Yel L, Roifman CM, Gardulf A, Eichmann F, Haag S, Massion C, Ochs HD. Healthrelated quality of life and treatment satisfaction in North American patients with primary immunedeficiency diseases receiving subcutaneous IgG self-infusions at home. J Clin Immunol. 2006;26:65-72.

13. Burks AW, Sampson HA, Buckley RH. Anaphylactic reactions after gamma globulin administration in patients with hypogammaglobulinemia. Detection of IgE antibodies to IgA. N Engl J Med. 1986;314:560-4. 